\title{
Effects of socio-economic and environmental factors on the spatial heterogeneity of dengue fever investigated at a fine scale
}

\author{
Yubing Qu, ${ }^{1,2}$ Xun Shi, ${ }^{3}$ Yong Wang, ${ }^{1,3}$ Rendong $\mathrm{Li}^{2}$ Liang Lu, ${ }^{4}$ Qiyong $\mathrm{Liu}^{4}$ \\ ${ }^{1}$ State Key Laboratory of Resources and Environmental Information System, Institute of Geographical \\ Sciences and Natural Resources Research, Chinese Academy of Sciences, Beijing, China; ${ }^{2}$ Institute of \\ Geodesy and Geophysics, Chinese Academy of Sciences, Wuhan, Hubei, China; ${ }^{3}$ Department of Geography, \\ Dartmouth College, Hanover, NH, USA; ${ }^{4}$ National Institute for Communicable Disease Control and \\ Prevention, Chinese CDC, Beijing, China
}

\begin{abstract}
The spatial pattern of dengue fever cases is the result of complex interactions between the virus, the host and the vector, which
\end{abstract}

\footnotetext{
Correspondence: Yong Wang, State Key Laboratory of Resources and Environmental Information System, Institute of Geographical Sciences and Natural Resources Research, Chinese Academy of Sciences, A11, Datun Road Anwai, Beijing 100101, China.

Tel.: +86.010.6488.8179.

E-mail: wangy@igsnrr.ac.cn
}

Key words: Dengue; Fine scale; Spatial heterogeneity; Generalized additive model; China.

Contributions: YQ and XS contributed equally as first authors. YQ, YW, RL conceived and designed the experiments. YQ, XS, YW performed the experiments. YQ, YW, RL, LL, QL analyzed the data. YQ, $\mathrm{XS}, \mathrm{YW}, \mathrm{LL}, \mathrm{QL}$ contributed reagents/materials/analysis tools. YQ, $\mathrm{XS}, \mathrm{YW}$ wrote the paper.

Conflict of interest: the authors declare no potential conflict of interest.

Funding: this study was partially funded by the Strategic Priority Research Program of the Chinese Academy of Sciences (XDA19040103), the Ministry of Science and technology of China (2016YFC1201301) and the China Scholarship Council fund (201704910131). The funders had no role in study design, data analysis, decision to publish, or preparation of the manuscript, which exclude data collection and laboratory test.

Ethical statement: the study protocol was approved by the Ethics Review Committee of the Chinese Center for Disease Control and Prevention, Beijing, China, the Guangdong Provincial Center for Disease Control and Prevention, Guangzhou, China. Written informed consent had also been obtained.

Received for publication: 6 March 2018 .

Revision received: 25 August 2018.

Accepted for publication: 3 October 2018.

(C) Copyright Y. Qu et al., 2018

Licensee PAGEPress, Italy

Geospatial Health 2018; 13:682

doi:10.4081/gh.2018.682

This article is distributed under the terms of the Creative Commons Attribution Noncommercial License (CC BY-NC 4.0) which permits any noncommercial use, distribution, and reproduction in any medium, provided the original author(s) and source are credited. may be affected by environmental conditions. The largest outbreak of dengue fever in Guangzhou city, China occurred in 2014 with case numbers 2.7 times the number of cumulative cases since 1978 and a significantly non-random spatial distribution. Selecting Guangzhou City as the study area, we used scan statistics to analyze the spatial heterogeneity of dengue fever and a generalized additive model to evaluate and examine the effects of socio-economic and environmental factors on spatial heterogeneity at a fine scale. The study found that the spatial distribution of dengue fever is highly heterogeneous and various factors differ in relative importance. The junction of the central districts of Guangzhou is a high-risk area with the urban village and urbanrural fringe zone formed by urbanization as important regional factors. The low gross domestic product per capita, the high population density, the high road density were perceived as risk factors. The Asian subtropical coastal area together with the socioeconomic and environmental factors were found to be the key drivers at the fine scale explaining the high spatial heterogeneity of dengue fever in Guangzhou City.

\section{Introduction}

Dengue fever is a mosquito-borne viral disease that occurs mainly in tropical and subtropical regions. According to World Health Organization, its incidence has increased by 30 times over the past 50 years and is now estimated that 390 million people worldwide are infected with dengue virus every year (Bhatt et al., 2013; Low et al., 2018; World Health Organization, 2018). The main vectors are Aedes aegypti and Ae. albopictus and the wide distribution of the latter has resulted in the spread of the wide dengue fever, particularly in Brazil and Argentina in the Americas, China and Indonesia in Asia as well as France and Portugal in Europe (La et al., 2010; Wilder-Smith et al., 2014). The rapid spread of dengue fever is the result of globalization, urbanization and ineffective vector control (Gubler, 2011; Low et al., 2018) as well as the lack of vaccine or drug treatment (Bhatt et al., 2013).

Thanks to application of geographical information systems in the public health area, the significant spatial heterogeneity of disease can be examined from a unique geographical perspective. At present, most of such research with respect to dengue use methods such as spatial autocorrelation (Ren et al., 2015) and scan statistics (Liu et al., 2014). However, the selection of scale in spatial autocorrelation analysis is subject to the subjective judgment of the researcher and does not consider the time characteristics of the aggregation, therefore easily resulting in incomplete detection of 
aggregation. Although scan statistic methodology effectively solves the bias problem of anthropomorphic scale, most of the research is based on large areas and large analysis units (nation, province, prefecture or county), so there is a lack of fine scale research in local areas. Therefore, this study used the scan statistics to elucidate the spatial heterogeneity of dengue fever in Guangzhou at a fine scale, i.e. the township level.

The temporal and spatial heterogeneity of dengue cases is related to geography, climate, and socioeconomic and environmental factors. These factors result in different patterns of aggregation (Wen et al., 2010; Ling et al., 2014) in space and time, and different patterns have different correlations with their related factors (Goto et al., 2013; Lai, 2011). Previous studies had many different foci. For example, Lowe et al. (2011) studied the effects of meteorological conditions on the risk of dengue fever in Brazil from 2001 to 2008, Wu et al. (2009) the influence of temperature and urbanization on the spatial distribution of dengue in Taiwan, Teurlai et al. (2015) the effects of socioeconomic and climatic factors on the heterogeneity of dengue fever on the island of New Caledonia from 1995 to 2012, while Fan et al. (2014) and other scholars considered the impact of meteorological factors on the spread of dengue fever in Guangdong Province from 2005 to 2011. These studies have explored the effects of various factors on dengue fever in different parts of the world, but the relative importance of individual factors may vary from country to region. Due to the low level of climate diversity in Guangzhou, socio-environmental factors may contribute to a large extent to the spatial heterogeneity of dengue fever, so we were only in studying the influence of socio-economic and environmental factors on spatial heterogeneity on a fine scale in Guangzhou, China.

Since 1997, there have been cases of dengue fever in China (Zhang et al., 2014; Fan et al., 2014). Between 2006 and 2014, these cases were mainly concentrated in Guangzhou, still an important region for dengue fever epidemics in China, which is also a key area for prevention and control. In 2014, the largest outbreak of dengue fever in southern China, a large proportion of cases $(83 \%)$ occurred in Guangdong Province. The objectives of this study were i) to analyze the spatial heterogeneity of dengue fever cases in Guangzhou based on the smallest administrative unit - the township (the hierarchal levels of Chinese administrative regions are province, prefecture, county and township with the county equivalent to district that exists both in rural and urban areas); and ii) to identify the socioeconomic and environmental factors that influence this spatial heterogeneity and examine and evaluate the mechanisms underlying each factor.

\section{Materials and Methods}

\section{Study area and background}

Guangzhou City is located at coordinates $22^{\circ} 26^{\prime} \sim 23^{\circ} 56^{\prime} \mathrm{N}$, $112^{\circ} 57^{\prime} \sim 114^{\circ} 03^{\prime} \mathrm{E}$. The climate is humid and subtropical, with high temperatures and humidity in summer with comparatively mild and dry winters. The annual mean temperature is $22^{\circ} \mathrm{C}$, and the annual accumulated precipitation is $1,800 \mathrm{~mm}$. The terrain is characterized by high elevation in the Northeast and low elevation in the Southwest. The North and Northeast are mountainous, with the maximum and average altitude of $1,210 \mathrm{~m}$ and $11 \mathrm{~m}$, respectively, while the South contains the Pearl River Delta alluvial plain. Meteorological conditions are suitable for the survival and repro- duction of the dengue virus, and the hot and humid climate is beneficial to the breeding and growth of mosquito vectors (Sang et al., 2015). In 2014, Guangzhou City experienced its most serious dengue epidemic, with 37,380 cases (four deaths), the greatest number of cases in any mainland Chinese city.

Guangzhou is a large city that has been at the forefront of China's reform and opening up, and it is the political, economic and cultural centre of Guangdong Province. Along with rapid urbanization, the rapid growth of the urban population, the expansion of urban villages and urban-rural fringe zones, high building density, widespread illegal construction, its complex population structure and hot and humid climate provide conditions for the outbreak of dengue fever (Zhu et al., 2012). China's urban villages are developed from rural settlements and become transitional neighbourhoods under rapid urbanization. The urban village is called chengzhongcun in Chinese, literally village encircled by the city, which was developed as a concept of a small urban settlement. It is of neighbourhood size combining residential living with work, retail and leisure units, aiming to be self-sustaining, mixing different social and economic groups, having efficient transport and is well designed and managed (Liu et al,. 2010). Urban-rural fringe zones can be seen as a transitional neighbourhood with a mixture of rural and urban society and dual land ownerships. It is a product of rapid urbanization and economic growths, and the dual urban and rural systems in terms of land ownership, household registration and planning management systems. According to the literature (Lin et al., 2012), there is a total of 138 urban villages in the metropolitan area with a total area of $80.6 \mathrm{~km}^{2}$, mainly located in the middle of the city of 63 townships. Urban-rural fringe areas are located at the junction of urban and rural areas with 35 townships (Lin et al., 2012). The incidence of dengue fever in Guangzhou continues to be is high and the prospects for prevention and control are not the best. These prospects are largely affected by geography, the natural ecological environment, climate characteristics, and social changes (increasing migrant, large population flow, etc.) Therefore, it is important to study the effect of social economy and environmental factors on the spatial heterogeneity of dengue fever in Guangzhou (Figure 1).

\section{Data collection}

\section{Administrative division data}

The data for the administrative divisions of the study area were retrieved from the Resources and Environment Science Data Center of the Chinese Academy of Science, including four levels of province, prefecture, county and township, which have previously been widely used in scientific research (Sang et al., 2016; Xiao et al., 2016).

\section{Dengue fever data}

Dengue fever cases were obtained from the Chinese Center for Disease Control and Prevention (China CDC) in 2014. Each case record consists of a patient's residential addresses and occupation, as well as the time of onset and diagnostic information. The inclusion criteria in our study included laboratory-confirmed or clinical diagnosis (based on clinical manifestations and epidemiologic exposure history), date of dengue fever onset in 2014 and permanent residential addresses within Guangzhou City. Spatial information technology was used to locate and calibrate each permanent address to ensure accuracy in the case location. 


\section{Socioeconomic and environmental data}

i) Urbanization. The impact of urbanization on the incidence of dengue fever can be described by two regional factors: urban village and urban-rural fringe zone, and one population factor, population density (Wu et al., 2009; Arunachalam et al., 2010). The above data were retrieved from the Resources and Environment Science Data Center of Chinese Academy of Science.

ii) Economic situation. The gross domestic product (GDP) of the counties in Guangzhou in 2015 was retrieved from the National Bureau of Statistics of China. We used a land-use model to analyze the gross domestic product of each township and further calculated the GDP per capita.

iii) Natural environment. The normalized difference vegetation index (NDVI) is an important indicator for assessing whether the target area contains green vegetation. The NDVI is in the range of -1 to +1 , where a high negative value indicates that the area has dense green vegetation. In the study, high-resolution, remote-sensing satellite images from 2014 were used. The NDVI values were calculated by band calculation, and the regional NDVI was obtained by regional statistical analysis.

iv) Accessibility. According to the literature, road density is an important index indirectly reflecting regional accessibility
(Mahabir et al., 2012). Roads are usually divided into five levels according to their administrative and utility classification, i.e. highway and road classes 1-4 (where class 1 is the best). The expressway runs mainly in the periphery of the city, so this paper used Class 1-4 roads from 2015 to calculate the road density, and regional statistical analysis was used to obtain the average road density for each township. Road data were obtained from the Resources and Environment Science Data Center of Chinese Academy of Science.

\section{Methods}

\section{Space-time scan statistical analysis}

Space-time scan statistics have been applied previously in the study of several mosquito-borne diseases such as malaria (Coleman et al., 2009), Japanese encephalitis (Impoinvil et al., 2011) and Barmah Forest virus (Naish and Tong, 2014). To identify factors affecting the distribution of outbreaks, space-time scan statistics are more accurate compared to purely spatial scans, as they assess two dimensions simultaneously, avoiding multiple test bias. We used the space-time scan statistics to test for the presence of statistically significant spatial and temporal clusters of dengue,

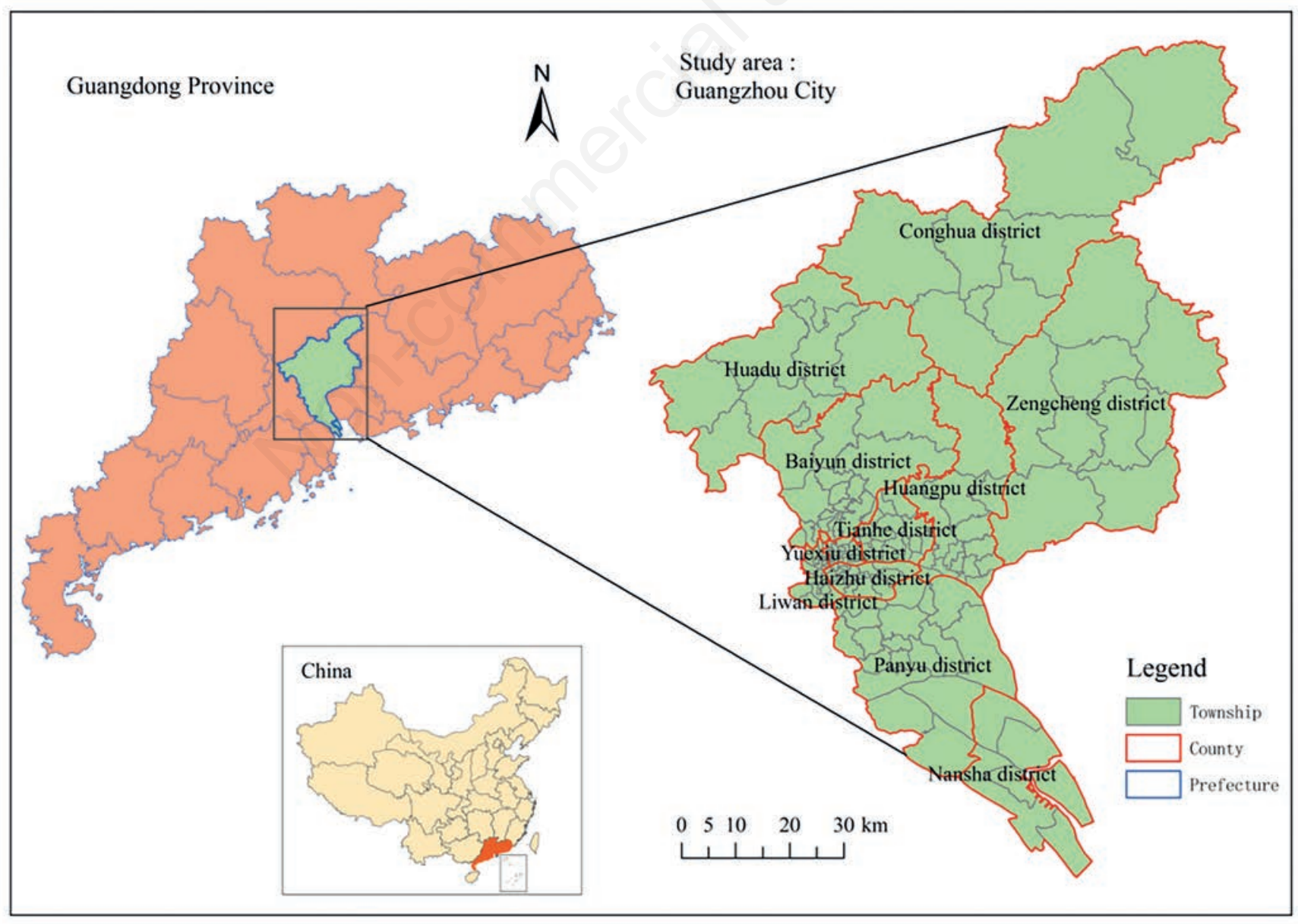

Figure 1. Study area. 
with the township as the scale of aggregation employing a cylindrical window with a circle indicating a geographic base with height representing the period of the potential clusters. The cylindrical window was then moved in space and time, to examine each potential geographical location and size, as well as each likely period. In addition, risk population is a population threshold limiting the size of the window. Each circle was noted as a potential cluster. Finally, for each potential cluster, a likelihood-ratio test statistic was used to determine whether the number of observed dengue cases within the potential cluster was significantly higher than expected. Space-time scan statistical analysis was performed using SaTScan v9.4.4 (Kulldorff, 2009).

\section{Generalized additive model analysis}

The generalized additive model (GAM) is a combination of the generalized linear model (GLM) and the additive model as it adds nonparametric smoothing terms based on GLM. The conditions for using GAM are as follows: i) each function can be summed and smoothed at the same time and; ii) the relationship between the independent variables and the dependent variables in the model is nonlinear with the data obeying the Poisson distribution, binomial distribution, gamma distribution or normal distribution. The general model is as follows (Eq. 1):

$$
g(\mu)=\alpha+\sum_{j=1}^{p} f_{j}\left(X_{j}\right)
$$

where $g(\mu)$ is a connection function and $f_{j}()$ a nonparametric smoothing function. The degree of freedom is an important index that influences the goodness of fit. The Akaike Information Criterion (AIC) criterion and the Generalized Cross Validation criterion are usually used as the criteria for the selection of the goodness of fit. To evaluate the possible non-linearity of the socioeconomic and environmental factors regarding the spatial heterogeneity of dengue, we used a spline-smoothing technique to fit and plot the predicted values based on the observed covariable values (Honorio et al., 2009). We specified the expected number of cases as follows (Eq. 2):

$\log ($ case $)=\beta_{0}+\beta_{1}$ (urban_village $)+\beta_{2}$ (urban_rural_fringe_zone $)+$ s(pop_density)+s(GDP_per_capita)+

s(NDVI)+s(road_density)

Eq. 2

where $\mathrm{s}()$ is the spline-smoothed, non-parametric function. The urban village and urban-rural fringe zone variables were binary, which fit with the parameter function. Population density, GDP per capita, NDVI and road density were nonlinear and fit with the spline-smoothed technique using specific degrees of freedom (df) for each smoothing. All statistical analyses were performed using the statistical software R 3.0.3 (Team, 2013) with the mgcv library (Wood, 2008).

\section{Results}

\section{Spatial distribution of dengue fever incidence}

Figure 2 shows the geographic distribution of targeted dengue fever incidence at the township level in Guangzhou. The colour change from green to red indicates increasing dengue fever incidence. Overall, the incidence of dengue fever in Guangzhou had high spatial heterogeneity. The high incidence was mainly distributed in particular townships in the middle of the city. There are 164 townships in Guangzhou, with the incidence of more than $1 \%$ $(100 / 10,000)$ in 24 townships. These townships include seven townships in the south-western Baiyun District, six townships each in the north-eastern Liwan District and north-western Haizhu District, two townships each in the western Tianhe District and Yuexiu District, and only one township in the north-eastern Panyu District. The total area is $138.83 \mathrm{~km}^{2}$, accounting for $1.93 \%$ of the city area.

\section{Spatial heterogeneity}

We used comparative sensitivity analysis and the Knox test to determine the maximum spatial window and the minimum time window of the scanning analysis to ensure the practical significance of the estimation of the spatial heterogeneity. Supported by previous studies and multiple data tests in study area, we identified minimum temporal windows, minimum spatial windows and maximum spatial windows for 14 days, $10 \%$ risk population, and $40 \%$ risk population, respectively. Thus, that means that one analysis window or one cluster area contains more than 14-day cases, more than $10 \%$ residents or less than $40 \%$ residents. To more accurately define spatial heterogeneity, two situations, 14 days \& $10 \%$ risk population and 14 days \& $40 \%$ risk population, were applied to space-time analysis. The intersection of the two analysis results was used as the final result, which was helpful to eliminate the error introduced by the circular aggregation window in the spacetime analysis.

The results of the analysis are shown in Table 1 and Figure 3. Figure $3 \mathrm{~A}$ shows the results of the first case: seven clusters, with a range of 85 townships. According to the log-likelihood ratio, it can be divided into a primary cluster and secondary clusters (Table 1). The results showed that the primary cluster is the most likely statistically significant cluster $(\mathrm{RR}=26.46)$, including 38 townships within the period $08 / 31 / 2014$ to $11 / 01 / 2014$. Another statistically significant secondary cluster with six circles was also identified within the same time frame (Figure 3B). Likewise, the primary cluster is the most likely statistically significant cluster $(\mathrm{RR}=42.32)$, including 102 townships within the period $08 / 31 / 2014$ to $10 / 29 / 2014$.

Although the sizes of the maximum spatial windows are different, the results of the space-time analyses were very similar (Figure 3C). The agreement of the two results in terms of time and the region were the most statistically significant clusters. The period of the cluster is from $08 / 31 / 2014$ to $10 / 01 / 2014$, which is consistent with the high-risk period from previous years (September to October). The overlapping areas included 67 townships mainly located at the junction of Baiyun District, Yuexiu District and Liwan District; the junction of Baiyun District, Tianhe District, Huangpu District the junction of Yuexiu District, Haizhu District and Tianhe District; and the junction of Haizhu District and Panyu District. The results showed that the high-risk period in Guangzhou was from September to October. There is a high incidence rate in the central area of the city, and the low incidence in other regions is a clear example of spatial heterogeneity. Human activities resulted in a lower incidence of disease inside each district than in the border areas. For a detailed analysis, see the Discussion section. 


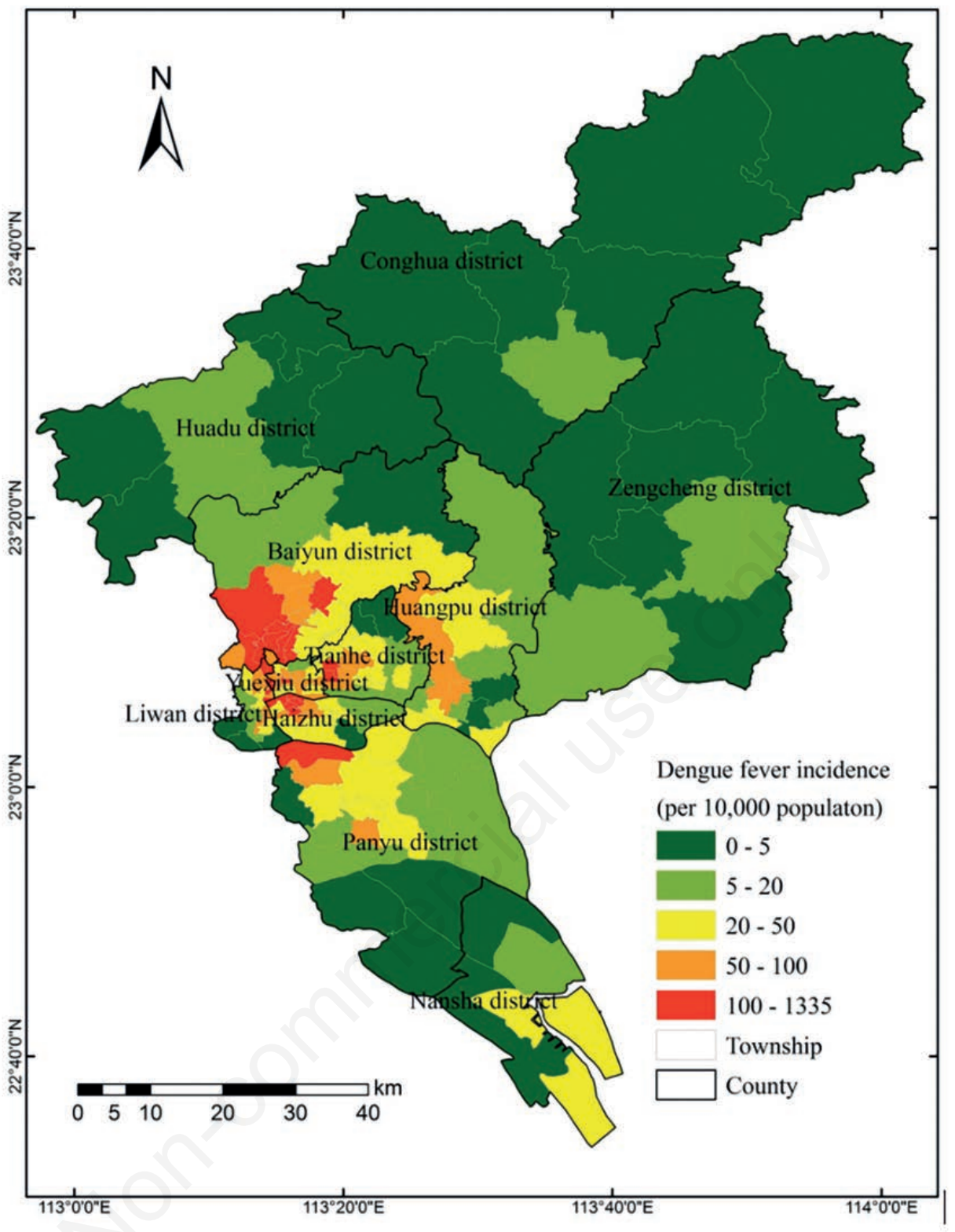

Figure 2. The spatial distribution of dengue fever incidence at the township level.

Table 1. Space-time clusters of dengue identified using space-time scan statistics.

\begin{tabular}{|c|c|c|c|c|c|c|c|}
\hline Period (10\%) & $C(n)$ & $\mathrm{T}$ & $\mathrm{R}(\mathrm{km})$ & $0(n)$ & $E(n)$ & $\mathbf{R R}$ & LLR \\
\hline Primary cluster & $1(38)$ & 08/31-11/01 & 8.48 & 11006 & 580 & 26.46 & 23599 \\
\hline Secondary cluster & $\begin{array}{l}1(16) \\
2(13) \\
3(6) \\
4(10) \\
5(1) \\
6(1)\end{array}$ & $\begin{array}{l}09 / 09-10 / 17 \\
09 / 06-10 / 20 \\
09 / 18-10 / 26 \\
09 / 18-10 / 17 \\
09 / 18-10 / 14 \\
09 / 24-10 / 20\end{array}$ & $\begin{array}{c}4.68 \\
10.25 \\
11.53 \\
9.59 \\
0.00 \\
0.00\end{array}$ & $\begin{array}{c}4909 \\
2850 \\
2640 \\
2352 \\
127 \\
68\end{array}$ & $\begin{array}{c}333 \\
330 \\
314 \\
288 \\
19 \\
12\end{array}$ & $\begin{array}{l}16.83 \\
9.26 \\
8.96 \\
8.65 \\
6.71 \\
5.74\end{array}$ & $\begin{array}{c}8931 \\
3709 \\
3367 \\
2934 \\
133 \\
62\end{array}$ \\
\hline Period $(40 \%)$ & $C(n)$ & $\mathrm{T}$ & $\mathrm{R}(\mathrm{km})$ & 0 (n) & $E(n)$ & $\mathbf{R R}$ & LLR \\
\hline Primary cluster & $1(102)$ & 08/31-10/29 & 16.81 & 29531 & 3051 & 42.32 & 55445 \\
\hline
\end{tabular}

C, Cluster; (n), number of townships; T, Time frame; R, Radius; O, Observed cases; E, Expected cases; RR, Relative risk; LLR, Log-likelihood ratio. 


\section{The effects of socio-economic and environmental fac- tors on spatial heterogeneity}

Analyses were performed with GAM to fit parametric and nonparametric functions to the relationships between the response and predictors. According to the criteria for assessing the goodness of fit for the dengue cases (Pearson $\chi 2 / \mathrm{df}$ close to 1 ), the distribution of dengue cases was over-dispersed and fit the negative binomial function (Pearson $\chi 2 / \mathrm{df}=0.71$ ) best. Thus, the log-link function for a negative binomial distribution response was used.

The spatial distribution of the six exploratory variables is mapped in Figure 4. Figure 4A shows the distribution of urban villages; Figure 4B shows the distribution of urban-rural fringes zones; Figure 4C shows the population density; Figure 4D shows the distribution of GDP per capita; Figure 4E shows the NDVI distribution; and Figure 4F shows the road density. The method for negative binomial response data was used to decide which terms to include in the model. The AIC/un-biased risk estimator scores for the models were compared with and without the term. Finally, all six factors were entered into the model, and we specified an offset for the predictor. The diagnostic information of the GAM showed that except for population density, the variables were found to be significant at the 0.01 level. This specification of the model explained $70.7 \%$ of the variance in the dengue fever incidence.

The partial contributions of six covariates to the conditional probability of the risk of disease are shown in Figure 5. Figure 5A and $\mathrm{B}$ show that the relative risk of living in urban-rural fringe areas (estimate value $=0.672$ ) was slightly larger than that of living in urban villages (estimate value $=0.663$ ). Figure $5 \mathrm{~A}$ shows that the urban village area had a positive impact on the risk of disease transmission. Figure 5B shows that the risk of disease in the urbanrural fringe areas will increase. Figure $5 \mathrm{C}$ shows that there was no significant linear relationship between population density and risk. We still consider that population density a crucial factor in dengue research because those townships of higher population density have more patients suffering from the disease. Figure 5D shows a significant downward trend of the risk of disease when per capita GDP increases to 100,000 CNY (14,500 USD). The small fluctuations around the zero response of risk to GDP per capita between $100,000 \mathrm{CNY}$ (14,500 USD) and 600,000 CNY (87,000 USD) must be deemed a modest, stable response. The risk showed a decreasing and then increasing trend when the GDP per capita is between 600,000 CNY (87,000 USD) and 800,000 CNY $(126,000$ USD), followed by a rapid decline. Thus, the higher the GDP per capita, the lower the risk of disease. Figure 5E depicts a negative relationship between the risk of disease and NDVI. When NDVI exceeded 0.25 , the risk of disease decreased rapidly with increasing NDVI. The result indicates that higher NDVI can decrease the risk of disease. The risk of disease decreased gradually with increasing road density (Figure 5F) showing a curve trough at approximately 50, after which the response increased. Therefore, this result indicates that high area accessibility can increase the risk of disease.

\section{Discussion}

\section{The effects of climate factors on the temporal hetero- geneity of dengue fever}

The seasonal variation in dengue fever is a clear example of temporal heterogeneity. The incidence of dengue fever in Guangzhou is concentrated in summer and autumn, and the highrisk period is from September to October. Dengue fever is a climate-sensitive disease; temperature plays an important role in the growth and breeding of mosquitoes and the transmission of dengue virus. Previous studies have found that the development time of Ae. albopictus follows an inverted V-shape as the temperature increases. The optimum growth temperature is $29.74^{\circ} \mathrm{C}$; above
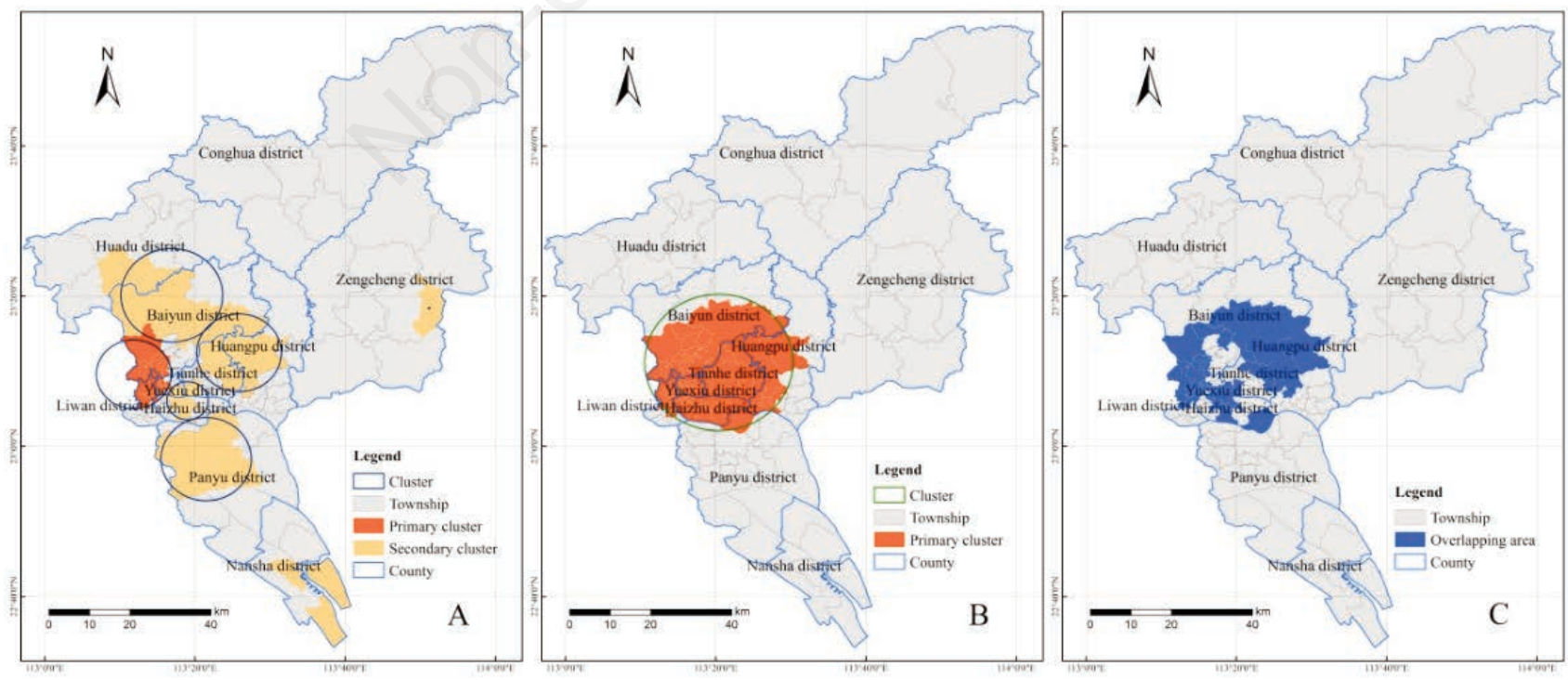

Figure 3. Space-time clusters of dengue identified using space-time scan statistics. (A) 14 days and $10 \%$ risk population; (B) 14 days and $40 \%$ risk population; (C) The area of agreement in analysis results. 

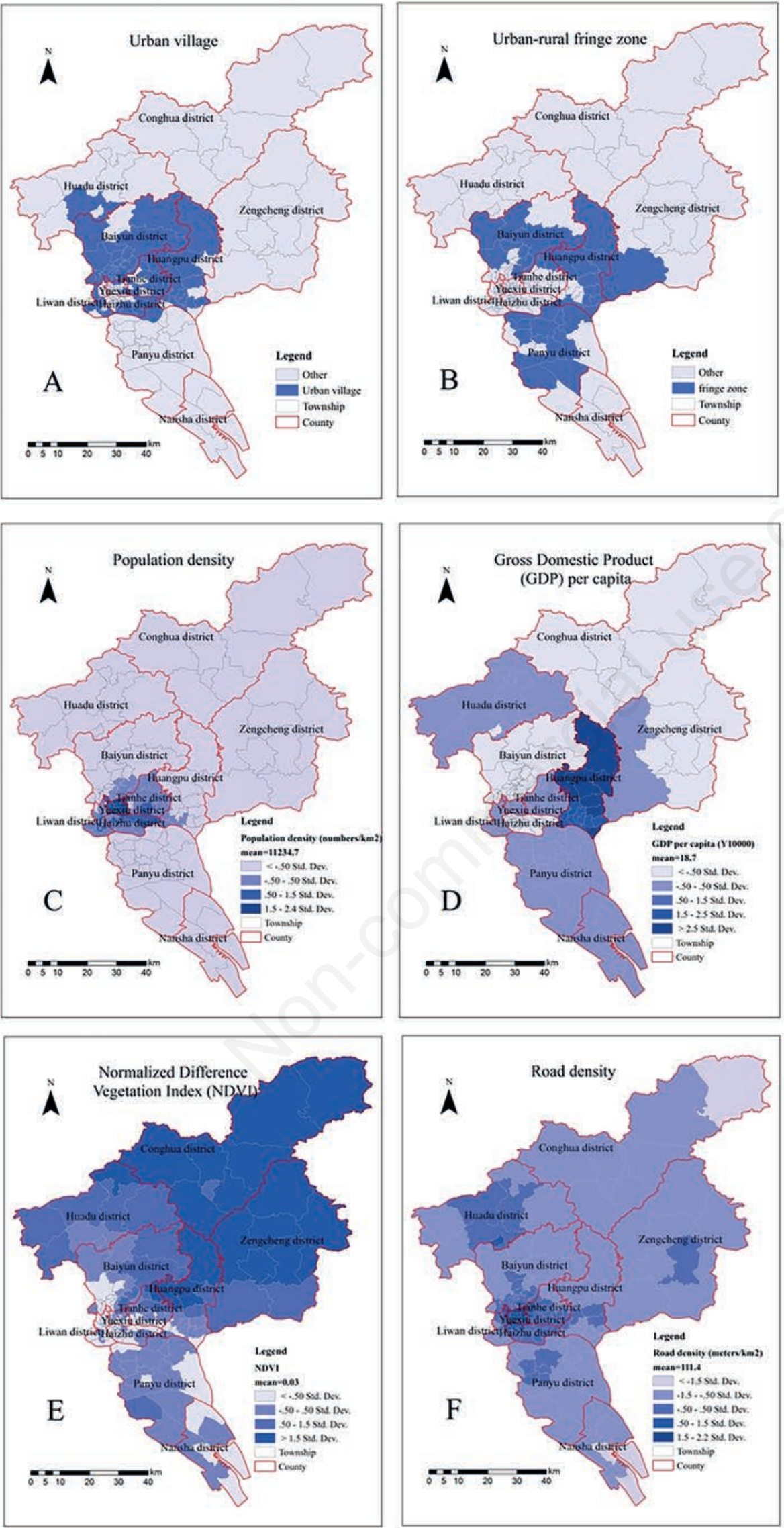

Figure 4. Spatial distribution of socioeconomic and environmental data at the township level. (A) Urban village; (B)

Urban-rural fringe zone; (C) Population density; (D) Gross Domestic Product per capita; (E)Normalized Difference Vegetation Index; (F) Road density. 
$30^{\circ} \mathrm{C}$, the development of the mosquito begins to be inhibited; and above $35^{\circ} \mathrm{C}$, only approximately $2.5 \%$ of the eggs develop into adults (Delatte et al., 2009). The transmission of dengue virus requires the development of the virus in the mosquito, which is called the extrinsic incubation period. Previous studies have presented that, as temperatures increase, the extrinsic incubation period is shortened between $18^{\circ} \mathrm{C}$ and $36^{\circ} \mathrm{C}$ and reaches a minimum at $31^{\circ} \mathrm{C}$ before increasing again. At temperatures lower than $18^{\circ} \mathrm{C}$, dengue virus cannot develop in mosquitoes (Xiao et al., 2016). These characteristics may explain the high incidence of dengue fever in Guangzhou in September and October. The results show that mosquito control should begin in May and June by promoting public awareness and cleaning up mosquito breeding grounds to keep mosquito density in the dengue epidemic period at a low
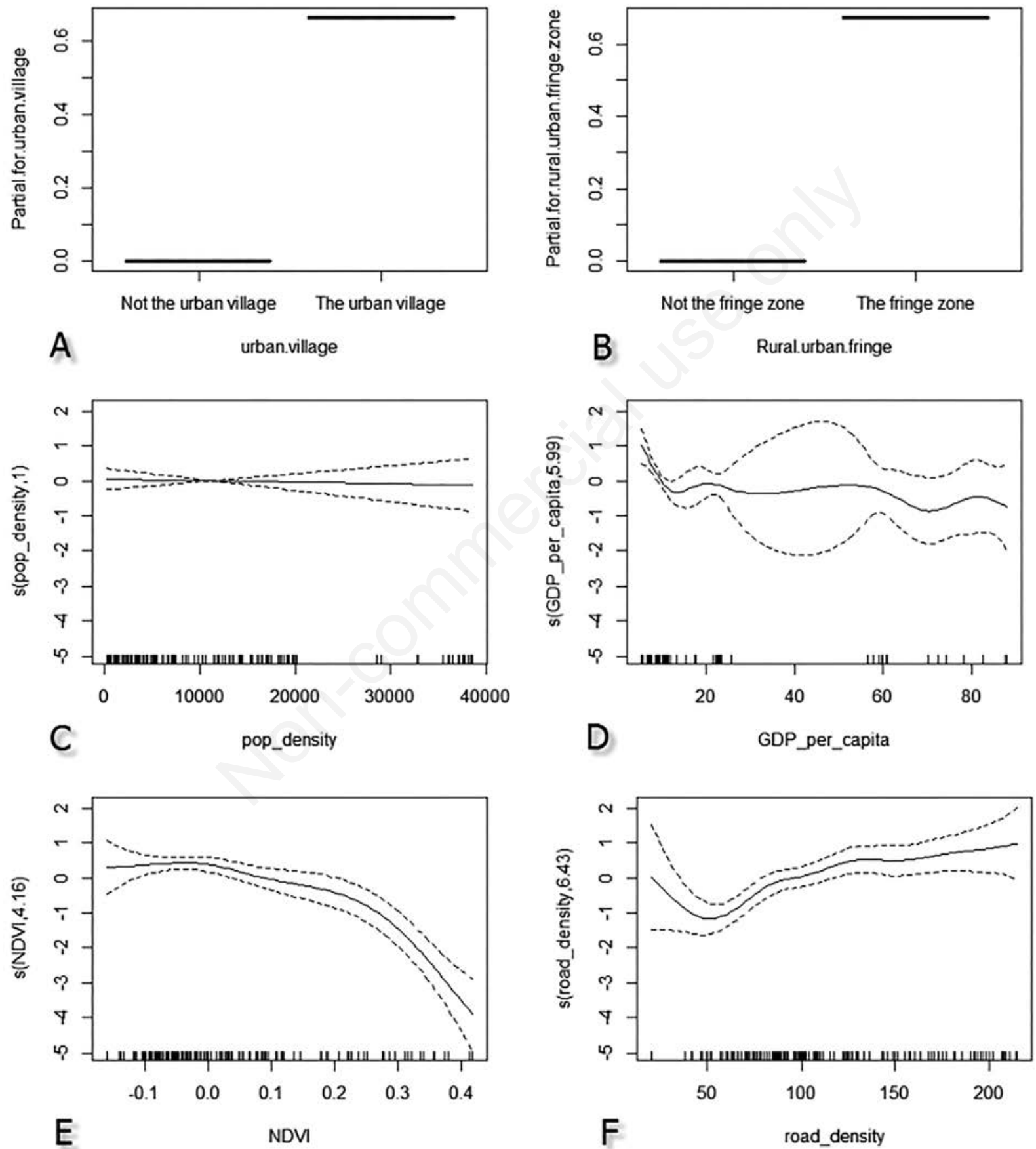

Figure 5. Partial contributions of six exploratory variables. (A) Urban village; (B) Urban-rural fringe zone; (C) Population density; (D) Gross Domestic Product (GDP) per capita; (E) Normalized Difference Vegetation Index (NDVI); (F) Road density. 
level. Climate factors, including temperature and precipitation, are often considered the major contributors to outbreaks of dengue (Wu et al., 2009; Wang et al., 2014), but they were not added to the GAM model because, according to the observation data of Guangzhou Meteorological Observatory, the spatial variation in temperature and precipitation was very small in 2014. Climate factors mainly affect the time heterogeneity of dengue fever, and rapid spread of dengue fever in a local area is more closely associated with socioeconomic and environmental factors than with climate (Lu et al., 2009; Kienberger et al., 2013; Teurlai et al., 2015). Additionally, the use of socioeconomic and environmental factors alone might increase the accuracy of the model.

\section{The choice of the size of the spatial window and tempo- ral window in space-time scan statistical analysis}

Space-time analysis can not only identify the aggregation area by spatial scanning but also automatically identify and filter the area in combination with temporal trends, which can improve the accuracy of detection. The choice of the size of the spatial window and temporal window determines the stability of the statistical analysis results, the accuracy of the cluster areas, and the operability of the disease monitoring work. It has been suggested that the maximum scan window for scanning statistical analysis in some areas seems unrealistic at the default setting of $50 \%$ of the risk population (Chen et al., 2008). Furthermore, landscape ecology is believed to strongly influence dengue transmission, and if a large population threshold ( $>40 \%$ ) were used, numerous functional ecological zones controlled by landscape ecology factors would be included in the area within that threshold. Thus, the most significant risk population thresholds were $10 \%$ and $40 \%$ in this study, based on the range tested by comparative sensitivity analysis (Naish and Tong, 2014).

In this study, the Knox test was used to detect the minimum cluster time of dengue fever. This test is one of the most commonly used statistical methods in the study of spatial interactions of infectious diseases, and it is usually used to detect the spatial and temporal distance distribution between cases of infectious diseases and diagnose the temporal and spatial spread of the disease with unknown causes and mechanisms (Knox and Bartlett, 1964; Kulldorff and Hjalmars, 1999). Aldstadt et al. (2012) used the Knox test to analyze 262 cases of dengue fever in Thailand from 1990 to 2010 and found that 15-17 days was the most likely continuous interval between cases. The latest research by Tao et al. (2016) analyzing 679 cases of dengue fever in the 20th-30th weeks of 2014 in Guangzhou using the Knox test, found that cases had significant spatial interactions at an interval of 2 weeks. Therefore, after a series of value tests, 14 days ( 2 weeks) was regarded as the minimum cluster time in space-time scan statistical analysis.

In this study, clusters generated using a larger size scan window (40\% risk population) produce larger clusters that could help policy-makers make decisions at larger geographic levels, for example, at the county level. However, these large spatial clusters cover a larger and more heterogeneous population. Conversely, clusters generated using smaller circular windows (10\% risk population) produce smaller clusters but contain a more homogeneous population, which could help policy makers plan more focused community interventions.

\section{The contribution of influencing factors}

The identification of socioeconomic and environmental factors and the partial trend shown in the model in this paper provide meaningful clues for epidemic assessment and local interventions to counteract risk factors. Compared with people in other regions in Guangzhou, people living in urban villages and urban-rural fringe areas are at higher risk of dengue infection. With the development of the city, the application of effective vector monitoring and control measures is important for reducing the risk of dengue fever. However, due to the rapid progress of urbanization and the lag in the development of management, cities, especially in the urban villages and urban-rural fringe areas, are facing an increasingly prominent sanitation problem; thus, the risk of infectious disease in these regions is increasing. Compared to previous studies (Qi et al., 2015), the same factors show different trends related to the risk of dengue fever in different regions. In Guangzhou, the vegetation index is negatively correlated with the risk of disease. As the vegetation index increases, the risk of disease diminishes, likely because in the park area, population density is low, vegetation coverage high and health management departments have taken measures to kill A. albopictus in the activity area. The GDP per capita shows a clear relationship to risk. The risk of dengue fever in low-income and high-income areas shows slight fluctuations, indicating that economic development is beneficial for reducing risk in both low-income and high-income areas. In urban areas with higher road density, the risk of disease increases as road density increases. We speculate that high accessibility maybe accelerate population flow and increase the rate of virus transmission. In contrast, in rural areas with low road density, the increase in road density is beneficial to improve the public environment somewhat and helps to reduce the risk of disease to a certain degree.

Human intervention can weaken or eliminate the impact of population density on the risk of dengue fever. GAM model analysis found that the effect of population density factors on the incidence of dengue fever is linear and non-significant. This factor should have been removed from this study, but population density is often considered to be one of the most important factors affecting dengue fever, and high-population-density regions usually have long epidemic times, high transmission speed and high morbidity. Therefore, it is essential to analyze why this factor was not significant. The reasons are as follows: there was a large-scale outbreak of dengue fever in Guangzhou in 2013, so the Patriotic Health Campaign strengthened the prevention and control measures taken in densely populated townships in 2014; these measures slowed the spread of the and reduced the incidence in the central region to below that in the border area in the counties with high population density. This finding was supported in our spatial heterogeneity analysis.

No significant relationship between population density and spatial heterogeneity of dengue fever was found in this study, but in areas of high population density, especially urban villages and urban-rural fringe areas, clean-up campaigns should be carried out ahead of time and intensified in the event of an epidemic to prevent outbreaks of dengue fever. We have taken many measures to verify the variable of population density by using smaller analysis units $(1 \mathrm{~km} \times 1 \mathrm{~km}$ grid) and adding other types of environmental variable and eventually prove that population density is a significant factor, and it's positively correlated with dengue risk. Low GDP per capita, low NDVI and high road density are the linear factors affecting the spatial heterogeneity of dengue fever, so environmental hygiene and $A$. albopictus eradication are still the focus of prevention and control work in urban areas. Devoting more resources to particularly vulnerable areas of the city will not only help to strengthen people's awareness of defense in their daily lives but 
also promote the implementation of timely response strategies in the early stages of dengue fever, thereby minimizing its epidemic potential.

This study is the first to identify the relationship between socioeconomic and environmental factors and the spatial heterogeneity of dengue fever in the important epidemic areas in mainland China. Spatial information technology was used to analyze the influence of socioeconomic and environmental factors on the spatial heterogeneity of dengue fever at a fine scale, which is the innovation of this research method. The extension of this study to other world cities that have experienced serious epidemics will help to raise awareness of the impact of environmental conditions on spatial heterogeneity at a fine level, and the identification of high-risk areas and influence mechanisms will also contribute to designing effective control measures for health authorities.

Regarding the link between socio-economic variables and dengue incidence rates during epidemics, a limitation of this study is the absence of historical time series of socio-economic and environmental variables. Some factors that could influence the spatial distribution of dengue cases during epidemics have not been taken into account in this study: the spatial variability in population immunity, or dengue vector control measures. The analysis of the spatial pattern of infectious diseases, in relation with socio-economic or environmental factors raises a number of methodological issues, such as the spatial scale of aggregation of the data. These issues need further attention in the future to increase the quality of spatial epidemiological and environmental studies.

\section{Conclusions}

Dengue fever in Guangzhou City shows obvious spatial heterogeneity. The cases were mainly concentrated in the junction townships of districts in the city centre. The space-time scan statistical method increases the accuracy of the estimation of the spatial and temporal heterogeneity of dengue fever.

Six factors (urban village, urban-rural fringe zone, population density, GDP per capita, NDVI and road density) are the core contributors to the spatial heterogeneity of dengue fever in Guangzhou, and these areas should be the next focus in the prevention and control of dengue fever.

The dengue fever epidemics in Guangzhou city show the same trend seen in Guangdong Province. Guangzhou is the highest incidence city in this province, and Guangdong province is the highest incidence region in mainland China, with dengue cases concentrated from July to November.

\section{References}

Aldstadt J, Yoon IK, Tannitisupawong D, Jarman RG, Thomas SJ, Gibbons RV, Uppapong A, Iamsirithaworn S, Rothman AL, Scott TW, Endy T, 2012. Space-time analysis of hospitalised dengue patients in rural Thailand reveals important temporal intervals in the pattern of dengue virus transmission. Trop Med Int Health 17:1076-85.

Arunachalam N, Tana S, Espino F, Kittayapong P, Abeyewickreme W, Wai KT, Tyagi BK, Kroeger A, Sommerfeld J, Petzold M, 2010. Eco-bio-social determinants of dengue vector breeding: a multicountry study in urban and periurban Asia. Bull World
Health Organ 88:173-84.

Bhatt S, Gething PW, Brady OJ, Messina JP, Farlow AW, Moyes CL, 2013. The global distribution and burden of dengue. Nature 496:504-7.

Chen J, Roth RE, Naito AT, Lengerich EJ, Maceachren AM, 2008. Geovisual analytics to enhance spatial scan statistic interpretation: an analysis of U.S. cervical cancer mortality. Int J Health Geogr 7:57.

Coleman M, Mabuza AM, Kok G, Coetzee M, Durrheim DN, 2009. Using the SaTScan method to detect local malaria clusters for guiding malaria control programmes. Malar J 8:68.

Delatte H, Gimonneau G, Triboire A, Fontenille D, 2009. Influence of temperature on immature development, survival, longevity, fecundity, and gonotrophic cycles of Aedes albopictus, vector of chikungunya and dengue in the Indian Ocean. J Med Entomol 46:33-41.

Fan J, Lin H, Wang C, Bai L, Yang S, Chu C, Yang W, Liu Q, 2014. Identifying the high-risk areas and associated meteorological factors of dengue transmission in Guangdong Province, China from 2005 to 2011. Epidemiol Infect 142:634-43.

Goto K, Kumarendran B, Mettananda S, Gunasekara D, Fujii Y, Kaneko S, 2013. Analysis of effects of meteorological factors on dengue incidence in Sri Lanka using time series data. PLoS One 8:e63717.

Gubler DJ, 2011. Dengue, urbanization and globalization: the unholy trinity of the 21(st) Century. Trop Med Health 39:3-11.

Honorio NA, Nogueira RM, Codeco CT, Carvalho MS, Cruz OG, Magalhaes, Mde A, 2009. Spatial evaluation and modeling of Dengue seroprevalence and vector density in Rio de Janeiro, Brazil. PLoS NeglTrop Dis 3:e545.

Impoinvil DE, Solomon T, Schluter WW, Rayamajhi A, Bichha RP, Shakya G, 2011. The spatial heterogeneity between Japanese encephalitis incidence distribution and environmental variables in Nepal. PLoS One 6:e22192.

Kienberger S, Hagenlocher M, Delmelle E, Casas I, 2013. A WebGIS tool for visualizing and exploring socioeconomic vulnerability to dengue fever in Cali, Colombia. Geospat Health 8:313-6

Knox EG, Bartlett MS, 1964. The detection of space-time interactions. Applied statistics 13:25-29.

Kulldorff M, 2009. SatScan ${ }^{\mathrm{TM}}$ Version 8.02: Software for the spatial and space-time scan statistics.

Kulldorff M, Hjalmars U, 1999. The Knox method and other tests for space-time interaction. Biometrics 55:544-52.

La Ruche G, Souares Y, Armengaud A, 2010. First two autochthonous Dengue virus infections in metropolitan France, September 2010. Euro Surveill 15:19676.

Lai LW, 2011. Influence of environmental conditions on asynchronous outbreaks of dengue disease and increasing vector population in Kaohsiung, Taiwan. Int J Environ Health Res 21:133-46.

Lin YL, de Meulder B, Wang SF, 2012. The interplay of state, market and society in the socio-spatial transformation of "villages in the city" in Guangzhou. Environ Urban 24:325-43.

Ling CY, Gruebner O, Krämer A, Lakes T, 2014. Spatio-temporal patterns of dengue in Malaysia: combining address and subdistrict level. Geospat Health 9:131-40.

Liu C, Liu Q, Lin H, Xin B, Nie J, 2014. Spatial analysis of Dengue fever in Guangdong Province, China, 2001-2006. Asia Pac J Public Health 26:58-66.

Liu Y, He S, Wu F, Wu F, Webster C, 2010. Urban villages under 
China's rapid urbanization: Unregulated assets and transitional neighbourhoods. Habitat Int 34:135-44.

Low G, Papapreponis P, Isa RM, Gan SC, Chee HY, Te KK, Hatta NM, 2018. Geographical distribution and spatio-temporal patterns of hospitalization due to dengue infection at a leading specialist hospital in Malaysia. Geospat Health 13:127-34.

Lowe R, Bailey TC, Stephenson DB, Graham RJ, Coelho CAS, Carvalho MS. Barcellos C, 2011. Spatio-temporal modelling of climate-sensitive disease risk: Towards an early warning system for dengue in Brazil. Comput Geosci 37:371-81.

Lu L, Lin H, Tian L, Yang W, Sun J, Liu Q, 2009. Time series analysis of dengue fever and weather in Guangzhou, China. BMC Public Health 9:395.

Mahabir RS, Severson DW, Chadee DD, 2012. Impact of road networks on the distribution of dengue fever cases in Trinidad, West Indies. Acta Trop 123:178-83.

Naish S, Tong S, 2014. Hot spot detection and spatio-temporal dynamics of dengue in Queensland, Australia. Int Archiv Photogramm Remote Sensing Spatial Inform Sci (ISPRS) XL8:197-204.

Qi X, Wang Y, Li Y, Meng Y, Chen Q, Ma J, Gao GF, 2015. The effects of socioeconomic and environmental factors on the incidence of Dengue fever in the Pearl River Delta, China, 2013. PLoS Negl Trop Dis 9:e0004159.

Ren H, Ning W, Lu L, Zhuang D, Liu Q, 2015. Characterization of dengue epidemics in mainland China over the past decade. J Infect Dev Ctries 9:970-6.

Sang S, Wang S, Lu L, Bi P, Lv M, Liu Q, 2016. The epidemiological characteristics and dynamic transmission of Dengue in China, 2013. PLoS Negl Trop Dis 10:e0005095.

Sang S, Yin W, Bi P, Zhang H, Wang C, Liu X, Jiang T, Wu H, Chu C, Liu Q, 2015. Predicting unprecedented dengue outbreak using imported cases and climatic factors in Guangzhou, 2014. Plos Negl Trop Dis 9:e0003808.

Tao H, Pan Z, Pan M, Zhuo L, Xu Y, Lu M, 2016. Mixing spatialtemporal transmission patterns of metropolis dengue fever: A case study of Guangzhou, China. Acta Geogr Sinica 71:165362.

Team RDC, 2013. R: A language and environment for statistical computing. R Foundation for Statistical Computing.
Computing 14:12-21.

Teurlai M, Menkès CE, Cavarero V, Degallier N, Descloux E, Grangeon JP, Guillaumot L, Libourel T, Lucio PS, MathieuDaudé F, Mangeaset M, 2015. Socio-economic and climate factors associated with Dengue fever spatial heterogeneity: a worked example in New Caledonia. PLoS Negl Trop Dis 9:e0004211.

Wang C, Jiang B, Fan J, Wang F, Liu Q, 2014. A study of the dengue epidemic and meteorological factors in Guangzhou, China, by using a zero-inflated Poisson regression model. Asia Pac J Public Health 26:48-57.

Wen TH, Lin NH, Chao DY, Hwang KP, Kan CC, Lin KC, Wu JT, Huang SY, Fan IC, King CC, 2010. Spatial-temporal patterns of dengue in areas at risk of dengue hemorrhagic fever in Kaohsiung, Taiwan, 2002. Int J Infect Dis 14:e334-43.

Wilder-Smith A, Quam M, Sessions O, Rocklov J, Liu-Helmersson J, Franco L, Khan K, 2014. The 2012 dengue outbreak in Madeira: exploring the origins. Euro Surveill 19:20718.

Wood SN, 2008. Fast stable direct fitting and smoothness selection for generalized additive models. J Royal Statist Soc: Series B (Statist Methodol) 70:495-518.

World Health Organization (WHO), 2018. Factsheet dated 13 September 2018. Available from: http://www.who.int/newsroom/fact-sheets/detail/dengue-and-severe-dengue Accessed: October 3, 2018.

Wu PC, Lay JG, Guo HR, Lin CY, Lung SC, Su HJ, 2009. Higher temperature and urbanization affect the spatial patterns of dengue fever transmission in subtropical Taiwan. Sci Total Environ 407:2224-33.

Xiao JP, He JF, Deng AP, Lin HL, Song T, Peng ZQ, Wu XC, Liu T, Li ZH, Rutherford S, Zeng WL, Li X, Ma WJ, Zhang YH, 2016. Characterizing a large outbreak of dengue fever in Guangdong Province, China. Infect Dis Poverty 5:44.

Zhang H, Zhang Y, Hamoudi R, Yan G, Chen X, Zhou Y, 2014. Spatiotemporal characterizations of dengue virus in mainland China: insights into the whole genome from 1978 to 2011. PLoS One 9:e87630.

Zhu YS, Breitung W, Li S, 2012. The changing meaning of neighbourhood attachment in Chinese commodity housing estates: evidence from Guangzhou. Urban Stud 49:2439. 